ARTICLE

\title{
Dynamic Europa ocean shows transient Taylor columns and convection driven by ice melting and salinity
}

Yosef Ashkenazy (iD ${ }^{1 凶} \&$ Eli Tziperman (i) ${ }^{2}$

The deep ( $100 \mathrm{~km})$ ocean of Europa, Jupiter's moon, covered by a thick icy shell, is one of the most probable places in the solar system to find extraterrestrial life. Yet, its ocean dynamics and its interaction with the ice cover have received little attention. Previous studies suggested that Europa's ocean is turbulent using a global model and taking into account nonhydrostatic effects and the full Coriolis force. Here we add critical elements, including consistent top and bottom heating boundary conditions and the effects of icy shell melting and freezing on ocean salinity. We find weak stratification that is dominated by salinity variations. The ocean exhibits strong transient convection, eddies, and zonal jets. Transient motions organize in Taylor columns parallel to Europa's axis of rotation, are static inside of the tangent cylinder and propagate equatorward outside the cylinder. The meridional oceanic heat transport is intense enough to result in a nearly uniform ice thickness, that is expected to be observable in future missions.

\footnotetext{
${ }^{1}$ Department of Solar Energy and Environmental Physics, The Blaustein Institutes for Desert Research, Ben-Gurion University of the Negev, Midreshet BenGurion, Negev 84990, Israel. ${ }^{2}$ Department of Earth and Planetary Sciences and School of Engineering and Applied Sciences, Harvard University, 20 Oxford

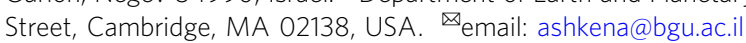


$\mathrm{T}$ he possibility of life outside Earth has long-fascinated humankind, and Europa, one of the four Galilean moons of Jupiter, is often mentioned as a candidate ${ }^{1-3}$ due to its deep $(\sim 100 \mathrm{~km})$ ocean ${ }^{4-6}$ that underlies a thick icy shell (several to tens of $\mathrm{kms})^{4,5,7-9}$ ). Europa has a relatively young surface ${ }^{10}$, indicating active ice shell tectonics ${ }^{5}$, and exhibiting chaotic terrain patterns $s^{4,11}$. The existence of an ocean under the icy shell is indicated by the observed induced magnetic field ${ }^{12}$, the indications of ice tectonics ${ }^{13}$ and perhaps also by water vapor plumes over Europa's mid-southern latitudes ${ }^{14,15}$.

Europa's ocean dynamics have been studied using a variety of models and mechanisms ${ }^{16-24}$. It has been suggested that localized ocean convection plumes may underlie the observed surface patterns of Europa ${ }^{16,17,22}$. On Earth, due to the very low oceanic aspect ratio (depth over horizontal scale, $\sim 10^{-3}$ ), only the vertical component of the Coriolis force is relevant. However, the aspect ratio of Europa's ocean is much higher $(\sim 1 / 16)$, and thus the horizontal components of the Coriolis force must be included and have been suggested to result in convection plumes that are parallel to the axis of rotation ${ }^{20,21,23,25}$. Scaling arguments were used to suggest the existence of alternating zonal jets ${ }^{20}$, and tidal forcing was proposed to lead to Rossby-Haurwitz waves and thus to oceanic tidal dissipation ${ }^{19}$. Tides can also excite internal waves ${ }^{26}$ and libration-driven elliptical instability can also drives ocean motions ${ }^{27}$. Recent studies of Europa's ocean ${ }^{23,25}$ used a global model, taking into account elements such as nonhydrostatic effects and the full Coriolis force, to study the ocean dynamics, and reported a wide low-latitude eastward jet, a high-latitude westward jet, and a rich eddy field. However, the model was adopted from core convection applications and therefore neglected salinity and ice freezing and melting effects that are shown below to dominate those of temperature; it also used upper and lower boundary conditions of prescribed temperature.

Here we show that a more self-consistent formulation, of prescribed bottom heat flux, and a top boundary condition that represents the full interaction with the icy shell and the resulting heat and fresh water fluxes, lead to a very different ocean temperature distribution. Our resolution is higher than that used previously by an order of magnitude, and the viscosity accordingly lower, allowing interesting small scale features to appear.

\section{Results}

The model. We use a very high-resolution ocean General Circulation Model (GCM), the MITgcm ${ }^{28,29}$ to investigate the ocean dynamics of Europa, first in a $2 \mathrm{~d}$ (latitude-depth) configuration, and then in a near pole-to-pole $3 \mathrm{~d}$ geometry. While the $2 \mathrm{~d}$ simulations lack several important physical processes, these simulations provide invaluable insight into several critical elements that cannot be addressed in $3 \mathrm{~d}$, mostly due to computational cost. We include all components of the Coriolis force, and use the full, non-hydrostatic dynamics. We use a prescribed heat flux as a boundary condition at the bottom rather than prescribing the temperature. This allows the temperature, and in particular the vertical temperature gradient (stratification) to be determined by the model. We follow the modern oceanographic literature and use a three equation formulation ${ }^{30}$ (see subsection "3-equation top boundary condition formulation") of the interaction between the icy shell and the ocean temperature and salinity fields, which takes into account the effects of freezing and melting of the icy shell, and diffusion of heat through the ice, on the temperature and salinity. The icy shell is assumed of uniform thickness, an assumption that we show below to be self-consistent with the calculated ocean meridional heat fluxes that were shown previously $^{31}$ to lead to a uniform ice thickness. Estimates of the mean salinity of Europa's ocean vary widely ${ }^{32}$, and we choose a value that is close to the lower end of estimates, of $50 \mathrm{ppt}(\mathrm{g} / \mathrm{kg})$. We later analyze the sensitivity to this choice.

2d Model results: stratification, salinity and Taylor columns. The 2d (latitude-depth) simulations (Fig. 1) show that the bottom geothermal heating results in a (potential) temperature at depth that is higher than near the ice-ocean interface by a mere $0.01^{\circ} \mathrm{C}$ (Fig. 1a), suggesting that the ocean is well mixed. In addition, note several interesting features. First, surprisingly, the coldest water is at low latitudes, despite the much warmer low-latitude ice surface temperature ${ }^{33,34}$, in contradiction to the findings of previous studies of Europa's ocean ${ }^{23,25}$. This is explained below as an effect of the Taylor columns. The ocean is stably stratified at high latitudes and unstable at low latitudes (Fig. 1c), as opposed to the globally unstable stratification imposed in the above mentioned previous studies. Water density variations are dominated by salinity variations, which dwarf the effects of temperature variations $(\beta \Delta S / \alpha \Delta T \gg 1$, where $\alpha$ and $\beta$ are the temperature and salinity expansion coefficients). Previous studies suggest that the salinity may in fact be even higher than assumed here ${ }^{32}$. In that case, the salinity gradients due to melting and freezing are expected to be even larger, as salinity rate of change is proportional to the fresh water forcing times the mean salinity (e.g., in the limit of a fresh ocean evaporation does not lead to salinity changes). We therefore focus on the sensitivity of our results to lower mean salinity values and show below that for a wide range of parameters the idea that salinity dominates density variations is robust (Supplementary Figs. 1-5). The zonal velocity (Fig. 2a, d) is westward in the low-latitude upper ocean and eastward elsewhere, with a typical velocity of a few $\mathrm{cm}$ per second. The deep equatorial zonal velocity is positive (eastward), indicating a superrotation, further discussed below.

Prominent arc-like structures appear in all fields (Figs. 1 and 2), which reflect features parallel to the rotation axis when plotted in spherical geometry (Fig. 2e). These are Taylor columns with ocean velocity nearly independent of the direction parallel to the rotation axis, and expected for an ocean with a nearly uniform density. While these columns were previously anticipated based on scaling arguments $^{20}$, simulated and attributed to convection ${ }^{25}$, and seen in simulations of magnetically-driven ocean circulation ${ }^{24}$, their detailed dynamics, structure, role in setting the large-scale temperature and salinity structure, and their spacing and propagation have not been studied.

The velocity along the Taylor columns fluctuates as one moves from Europa's center outward, between being positive and negative. Accordingly, the heat advection changes sign as well. In the region inside of the tangent cylinder that is aligned with the rotation axis and has the radius of Europa's rocky core, corresponding to latitudes less than about $20^{\circ 21}$, the columns intersect the ocean bottom and the ice base, and their alongcolumn motions effectively transfer the bottom geothermal heat to the ocean surface. However, outside the tangent cylinder, there is no such effective bottom-to-surface heat transport mechanism, as the Taylor columns do not intersect Europa's ocean bottom there, and this results in the colder ocean surface in the equatorial regime seen in Fig. 1a. This leads to freezing there, and thus to brine rejection and to the higher salinity as seen in Fig. $1 \mathrm{~b}$.

We find that the spacing between the Taylor columns is less than $20 \mathrm{~km}\left(0.75^{\circ}\right.$ latitude, Fig. 3a). In order to analyze the Taylor columns, we project the model's meridional $v$ and vertical $w$ velocity components on the directions parallel and perpendicular to the axis of rotation. The velocity parallel to the axis of rotation, $u_{\text {par }}=w \sin \phi+v \cos \phi$ where $\phi$ is the latitude, shows clear Taylor columns in which it is independent of the direction 

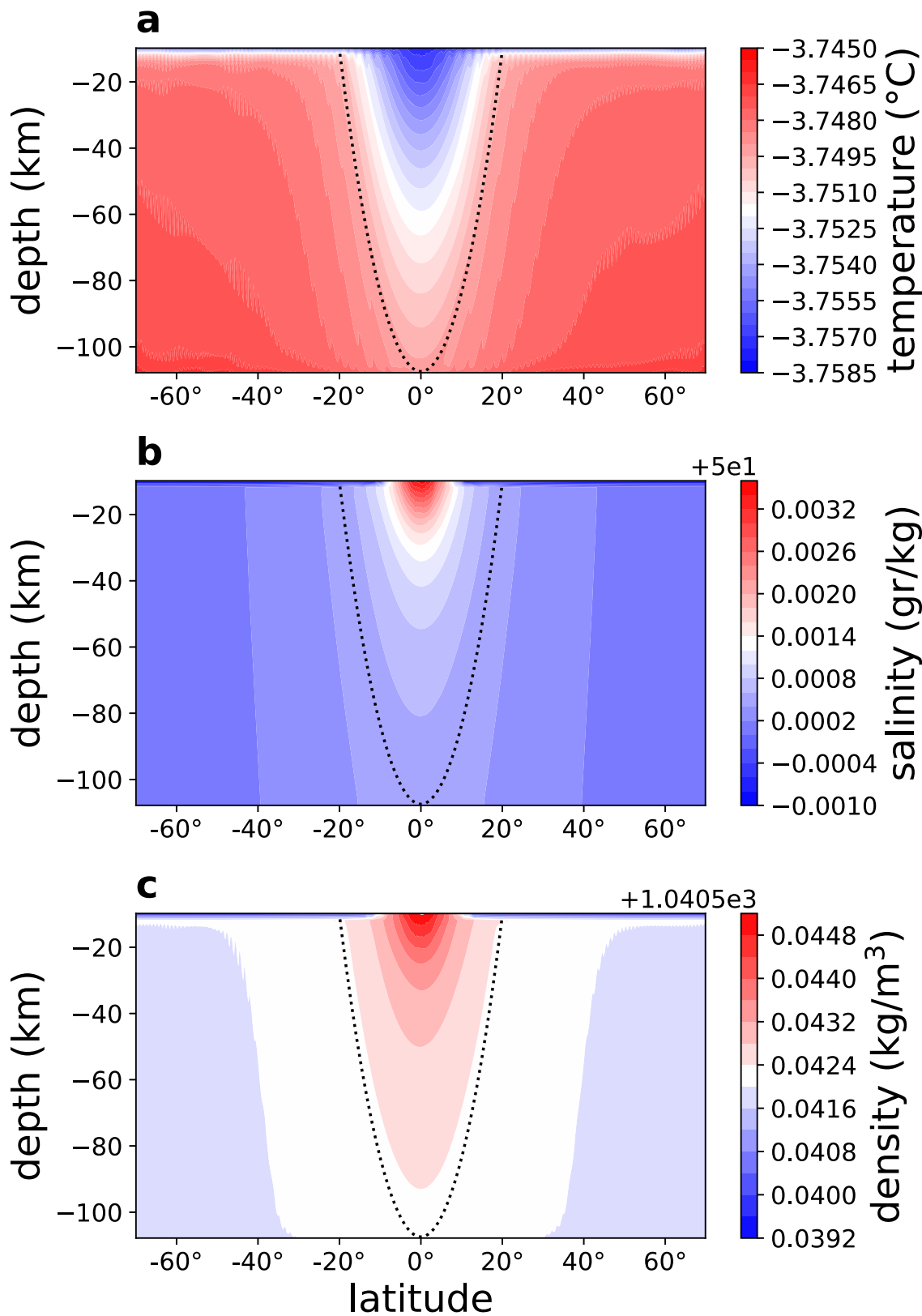

Fig. 12 d simulation results-tracers. Latitude-depth snapshot plots of a temperature $\left({ }^{\circ} \mathrm{C}\right)$, b salinity $\left(\mathrm{g} \mathrm{kg}^{-1}\right)$, and $\mathbf{c}$ density $\left(\mathrm{kg} \mathrm{m}^{-3}\right)$. The dotted lines in these panels shows the tangent cylinder.

parallel to the axis of rotation (Fig. $2 \mathrm{~b}$, e), in accordance with the Taylor-Proudman theorem. This parallel velocity is significantly smaller than the zonal velocity (Fig. 2a, d) and significantly larger than the velocity perpendicular to the axis of rotation in the latitude-depth plane $u_{\text {per }}=w \cos \phi-v \sin \phi$ (Fig. 2c). In the zonal momentum budget of the $2 \mathrm{~d}$ model, the two Coriolis terms dominate the others, so that the momentum balance is $2 \Omega w \cos \phi-2 \Omega v \sin \phi \approx 0$, where $\Omega$ is Europa's rotation rate. This leads to $u_{\text {per }} \approx 0$, explaining the observation that $u_{\text {per }} \ll u_{\text {par }}$. The parallel and zonal velocities are symmetric with respect to the equator, while the perpendicular velocity is anti-symmetric, vanishing at the equator.

The distance between the Taylor columns can be estimated using scaling arguments (see subsection "The spacing between the Taylor columns"). In the zonal and meridional dominant momentum balances, the sum of the two dominant Coriolis terms is balanced by parameterized horizontal viscosity, and one can form a length scale from the two relevant parameters, the horizontal viscosity coefficient $v_{\mathrm{h}}\left(\mathrm{m}^{2} \mathrm{~s}^{-1}\right)$ and the rotation rate $\Omega$ $\left(\mathrm{s}^{-1}\right)$, to find that the relevant length scale is $C \sqrt{\nu_{\mathrm{h}} \sin (\phi) / \Omega}$ where $C \approx 14$ is an empirical constant found from the numerical results (Fig. 3a). The horizontal viscosity coefficient we used (50 $\mathrm{m}^{2} \mathrm{~s}^{-1}$ ) represents parameterized viscosity (see subsection "Eddy coefficients, subgrid-scale representation"). Further verification of the scaling for the distance between columns is obtained below in the $3 \mathrm{~d}$ runs, where the effective resolved eddy viscosity is found to be larger $\left(300 \mathrm{~m}^{2} \mathrm{~s}^{-1}\right.$, see subsection "Estimating eddy coefficients"), and the column distance is indeed larger (Supplementary Fig. 6). While this particular spacing is likely sensitive to model assumptions, the qualitative dynamical insights obtained should be valid. Additional simulations suggest that the distance between the Taylor columns in the high latitudes scales like the square 

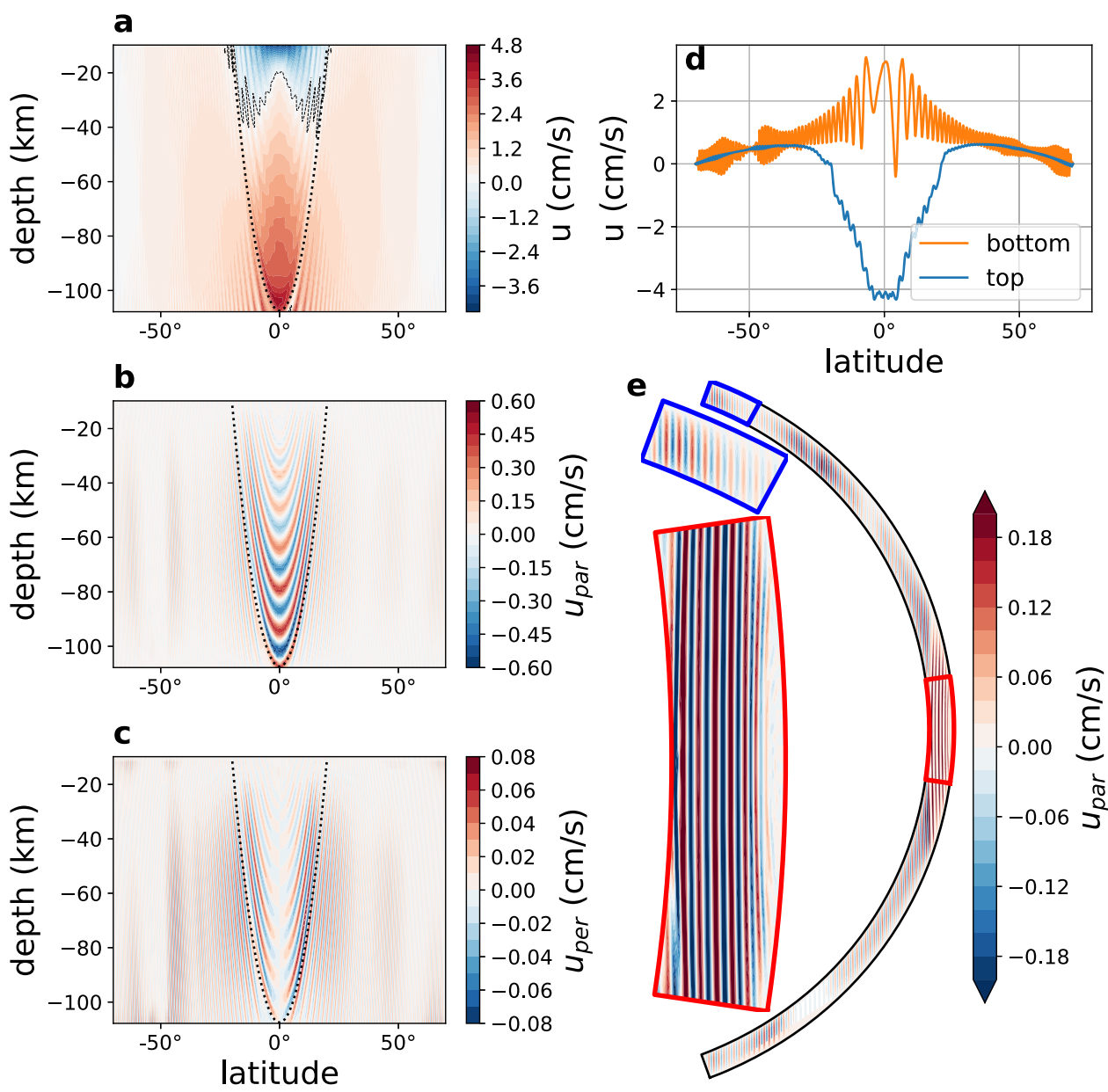

Fig. 2 2d simulation results-velocity. Latitude-depth snapshot plots of a zonal velocity ( $\mathrm{cm} \mathrm{s}^{-1}$, dashed line shows the zero contour), b velocity parallel to the axis of rotation, $u_{\mathrm{par}}\left(\mathrm{cm} \mathrm{s}^{-1}\right)$, and $\mathbf{c}$ velocity perpendicular to the axis of rotation, $u_{\mathrm{per}}\left(\mathrm{cm} \mathrm{s}^{-1}\right)$. The dotted lines in these panels shows the tangent cylinder. $\mathbf{d}$ Zonal velocity at the top and bottom of the ocean $\left(\mathrm{cm} \mathrm{s}^{-1}\right)$ as a function of latitude. $\mathbf{e}$ The spherical presentation of the velocity parallel to the axis of rotation $u_{\mathrm{par}}$ (also shown in $\mathbf{b}$ ), demonstrating Taylor columns that are parallel to the axis of rotation. The ocean depth extent is $100 \mathrm{~km}$ in all three frames shown, where the latitudinal extent of the main (black) frame is $70^{\circ} \mathrm{S}-70^{\circ} \mathrm{N}$, the low latitude (red) frame is $8^{\circ} \mathrm{S}-8^{\circ} \mathrm{N}$, and the high latitude (blue) frame is $61^{\circ} \mathrm{N}-70^{\circ} \mathrm{N}$.

root of the ocean depth. The existence of Taylor columns in Europa, and the corresponding zonal jet structure was predicted by ref. ${ }^{20}$ to be related to the Rhines scale, although our findings regarding the spacing between the columns is different from the Rhines scale scaling predicted in that work. These columns seem to also appear in one of the simulations of ${ }^{25}$ that was characterized by low viscosity, although no detailed analysis was provided.

In absence of dissipation the Taylor columns were predicted to be at a fixed latitude, as the potential vorticity $(q=(2 \Omega+\zeta) / h$, where $h$ is the column height, which depends on latitude) is preserved ${ }^{20}$. Yet we find the columns to show prominent equatorward propagation outside of the tangent cylinder (Fig. 3b, c) whose mechanism would require further elucidation in future work. No propagation is visible within the tangent cylinder (Fig. 3b, c). We also note the oscillatory variations along the maximum/minimum lines (Fig. 3b, c). While the discussion in this subsection clearly explains the structure and spacing of the (2d) Taylor columns, the necessarily-over simplified eddy viscosity formulation used may affect the simulation. Below we show, based on $3 \mathrm{~d}$ simulations, that in fact the eddy parameterized coefficient is much larger than the one used in the $2 \mathrm{~d}$ results, lending credibility to the $2 \mathrm{~d}$ results. 3d Model results: eddies, convecting plumes. We next consider a $3 \mathrm{~d}$ simulation of Europa's ocean at very high resolution (1/24 of a degree, compared with $\sim 1^{\circ}$ of previous studies ${ }^{23}$ ). The model spans $30^{\circ}$ longitude and we assume periodic boundary conditions in the zonal direction. The added 3rd, zonal, dimension allows for waves and eddies to develop and enables us to examine the interaction of eddies with the Taylor columns and convection (Figs. 4 and 5). The Taylor columns now appear most prominently in the simulated meridional and vertical velocity fields as isolated columns with a width and separation of about $20-50 \mathrm{~km}$ (Supplementary Figs. 7-11). The columns are again largely aligned with the rotation $\operatorname{axis}^{20,25}$ as in the $2 \mathrm{~d}$ model. At low latitudes, high-salinity downward convection plumes originate from the ice-ocean interface (Fig. 4b) due to brine-rejection during the freezing process that was not included in previous studies of Europa's ocean (upper part of Fig. 4b and Supplementary Videos 1 and 2). Upward plumes are seen in Fig. 4c to originate from the ocean bottom due to the geothermal heating there. These convective plumes are nearly perpendicular to the Taylor columns near the equator, in contradiction to expectations based on regional simulations ${ }^{20-22}$, and are also visibly advected by the mean zonal flows. The orthogonality of the low-latitude convection and Taylor columns suggests that these two classes of 

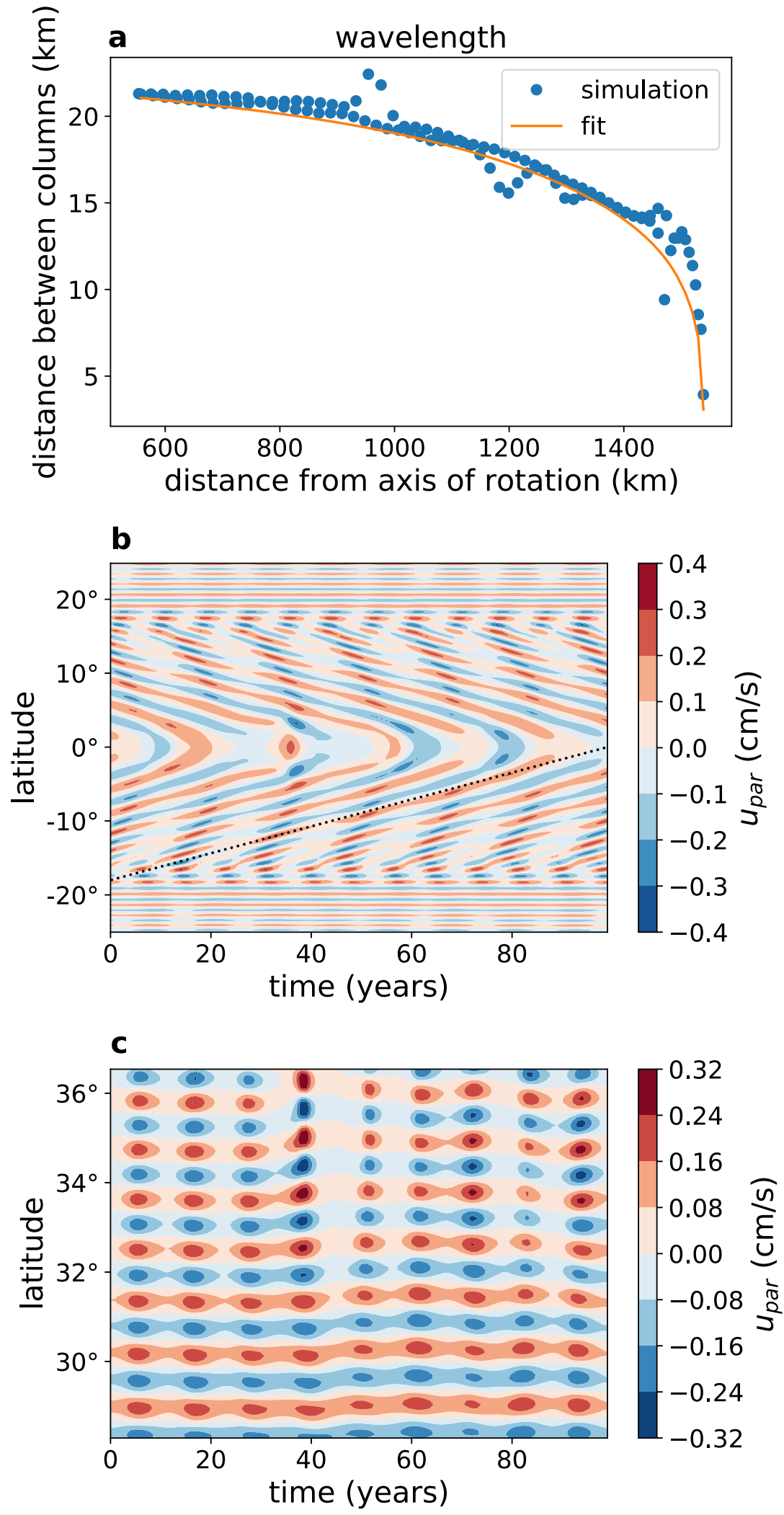

Fig. 3 The dynamics and structure of the Taylor columns. a The distance $(\mathrm{km})$ between the Taylor columns in the $2 \mathrm{~d}$ simulation as a function of the distance from the axis of rotation $(\mathrm{km})$, based on a snapshot of the velocity parallel to the axis of rotation, $u_{\text {par }}$ at a depth of $13 \mathrm{~km}$ under the ice-ocean interface. Full circles represent the numerically estimated distances while the solid line represents the functional fit, see text. $\mathbf{b}$ The velocity parallel to the axis of rotation $\left(\mathrm{cm} \mathrm{s}^{-1}\right)$ as a function of latitude and time (Earth years) at a depth of $19 \mathrm{~km}$ under the ice. The figure shows the equatorward propagation of the Taylor columns, where the slope of the shown dashed line corresponds to a propagation velocity of $0.18^{\circ}$ per year. c Same as $\mathbf{b}$, showing a region inside the tangent cylinder (at depth of $49 \mathrm{~km}$ under the ice), where the Taylor columns are static. 

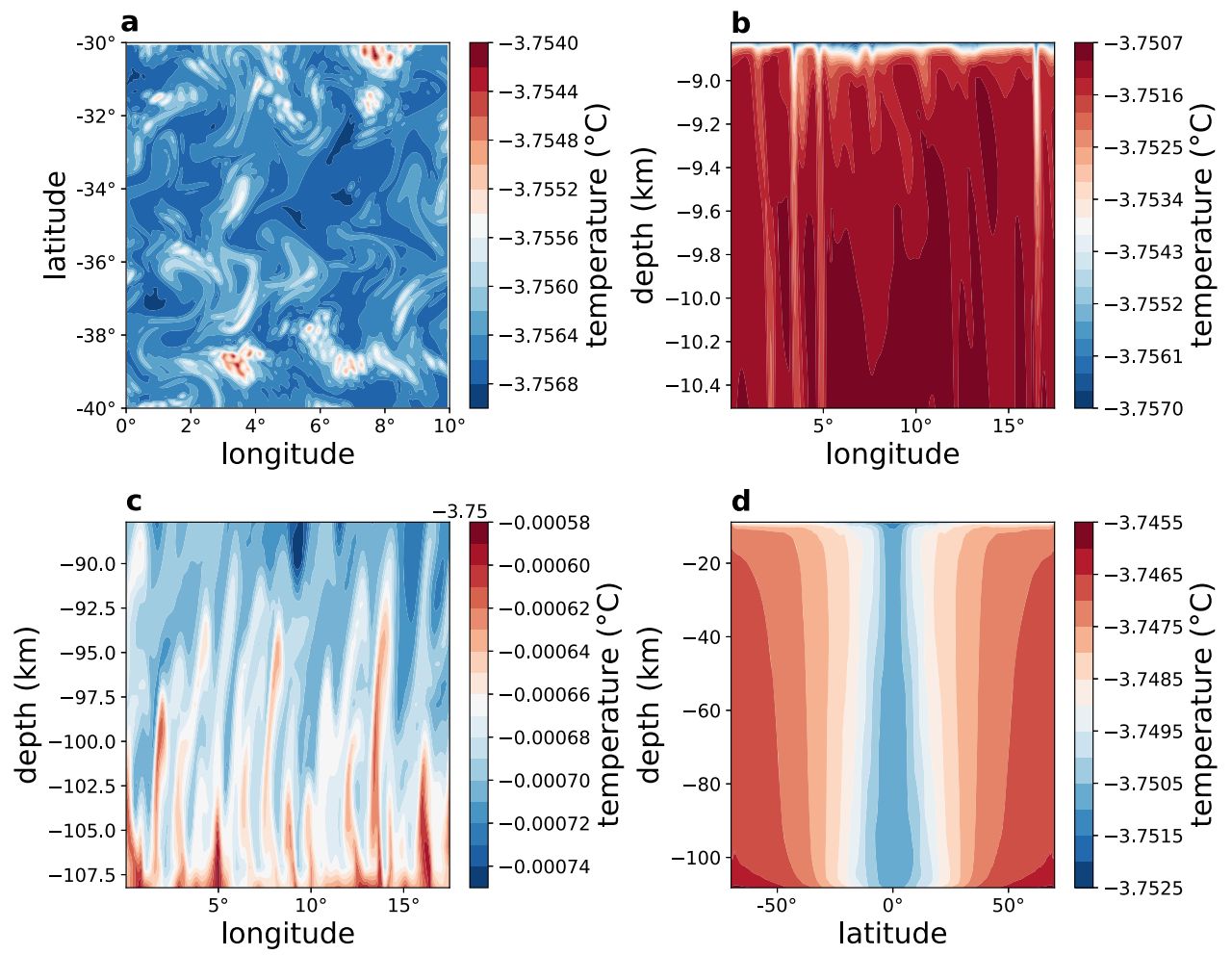

Fig. 4 Results of the 3d simulation-temperature. a Surface ocean temperature $\left({ }^{\circ} \mathrm{C}\right.$ ) as a function of longitude and latitude for a $10 \times 10^{\circ}$ region, showing a highly turbulent flow. b, c Depth-longitude temperature $\left({ }^{\circ} \mathrm{C}\right)$ sections near the top and bottom of the ocean at the equator, showing downward and upward convection plumes. At the equator, the local vertical (depth) direction is perpendicular to the Taylor columns, and the panels therefore demonstrate that convection does not necessarily occur along the Taylor columns. $\mathbf{d}$ Latitude-depth plots of zonal mean temperature $\left({ }^{\circ} \mathrm{C}\right)$.

motion are distinct. Furthermore, the $2 \mathrm{~d}$ sensitivity run shown in Supplementary Figs. 1-5 with a vanishing mean salinity shows a regime that is completely stratified with no convection occurring anywhere, yet with Taylor columns prominent in the zonal and meridional velocity components. The energy source for the Taylor columns, and in particular what is the specific instability mechanism involved, requires further study.

The temperature field is clearly turbulent, showing richly complex eddy filaments (Fig. 4a, see Supplementary Video 1). The above relation between viscosity and Taylor column spacing, together with the fact that the Taylor column spacing is larger in the $3 \mathrm{~d}$ simulation, suggests that the effective eddy viscosity due to resolved eddies is about 15 times larger than the small explicit parameterized viscosity used in the $3 \mathrm{~d}$ runs for numerical stability, following common ocean modeling practice. This is consistent with an explicit estimate of the eddy coefficients calculated from the $3 \mathrm{~d}$ runs (Supplementary Fig. 12). As a result of the eddies and convection plumes, the Taylor columns are less persistent along the direction parallel to the rotation axis than in the $2 \mathrm{~d}$ simulations (Figs. 4 and 5 and Supplementary Figs. 7-11). The existence of waves and eddies in the $3 \mathrm{~d}$ simulation also affects mean flows. The zonal velocity is typically several $\mathrm{cm} \mathrm{s}^{-1}$ (Fig. 5b), 1-2 orders of magnitude smaller than the previously reported velocities ${ }^{23,25}$; thus, our estimate for Europa's ocean kinetic energy (see below) is several orders magnitude smaller than that of these previous studies. Note in particular differences in the vertical structure of the zonal jets along the equator, between the $2 \mathrm{~d}$ (Fig. 2a, d) and $3 \mathrm{~d}$ (Fig. 5b) simulations; see also Supplementary Fig. 13. The 2d zonal flow shows superrotation only at depth, while the $3 \mathrm{~d}$ ones shows it at all depths. The $2 \mathrm{~d}$ vertical shear with superrotation at depth and a retrograde surface current is likely supported by an eddy flux of zonal momentum toward the rotation axis. The $3 \mathrm{~d}$ superrotation can be driven by Rossby waves that are possible in this configuration. Moreover, the increasing $2 \mathrm{~d}$ equatorial zonal velocity with depth is consistent with the thermal-wind relation (i.e., $\left.u_{z}=\frac{g}{2 \Omega a \rho_{0} \sin (\phi)} \rho_{\phi}\right)$. In the $3 \mathrm{~d}$ case, we find an additional term that cannot be neglected (i.e., $\left.a \sin (\phi) u_{z}+(\cos (\phi) u)_{\phi}=\frac{g}{2 \Omega \rho_{0}} \rho_{\phi}\right)$, explaining the different vertical structure of the zonal velocity $u$. The zonal domain of the model spans only $30^{\circ}$ for computational efficiency. However, because the typical size of eddies in the $3 \mathrm{~d}$ simulation is much smaller than the zonal extent, we expect the eddy dynamics to be similar had we considered a fuller zonal extent of $360^{\circ}$.

While the stratification is very weak and the ocean well-mixed (Fig. 1a-c), as in the $2 \mathrm{~d}$ case, the extent of unstable water column with heavy water above light water is more limited in the $3 \mathrm{~d}$ case (compare Figs. 1a-c, $4 \mathrm{c}$ and $5 \mathrm{a}$ ). This is because the eddies in the $3 \mathrm{~d}$ simulation strengthen the stratification, by converting potential energy into kinetic energy, as was suggested to be the case for Earth's snowball ocean ${ }^{35,36}$. The characteristic time of convection may be estimated via the buoyancy frequency, $N^{2}=-g / \rho_{0} \partial \rho /$ $\partial z \approx(g / H)\left(\Delta \rho / \rho_{0}\right)$ where negative/positive $N^{2}$ indicates statically unstable/stable water column. We find typical buoyancy and convection time scales, corresponding to positive and negative values of the buoyancy frequency, with corresponding time scales $2 \pi /|N|$ that both exceed 50 days. The positive values represent time scales of internal waves in Europa's ocean and are much larger than the corresponding time scales on Earth, and in fact represent an interesting regime where the Coriolis time scale is shorter than that of buoyancy oscillations. For negative values, the time scales are again much longer than those on Earth, and are consistent with the evolution times seen in the Supplementary Video chat 2 and with previous higher resolution regional runs ${ }^{22}$. 

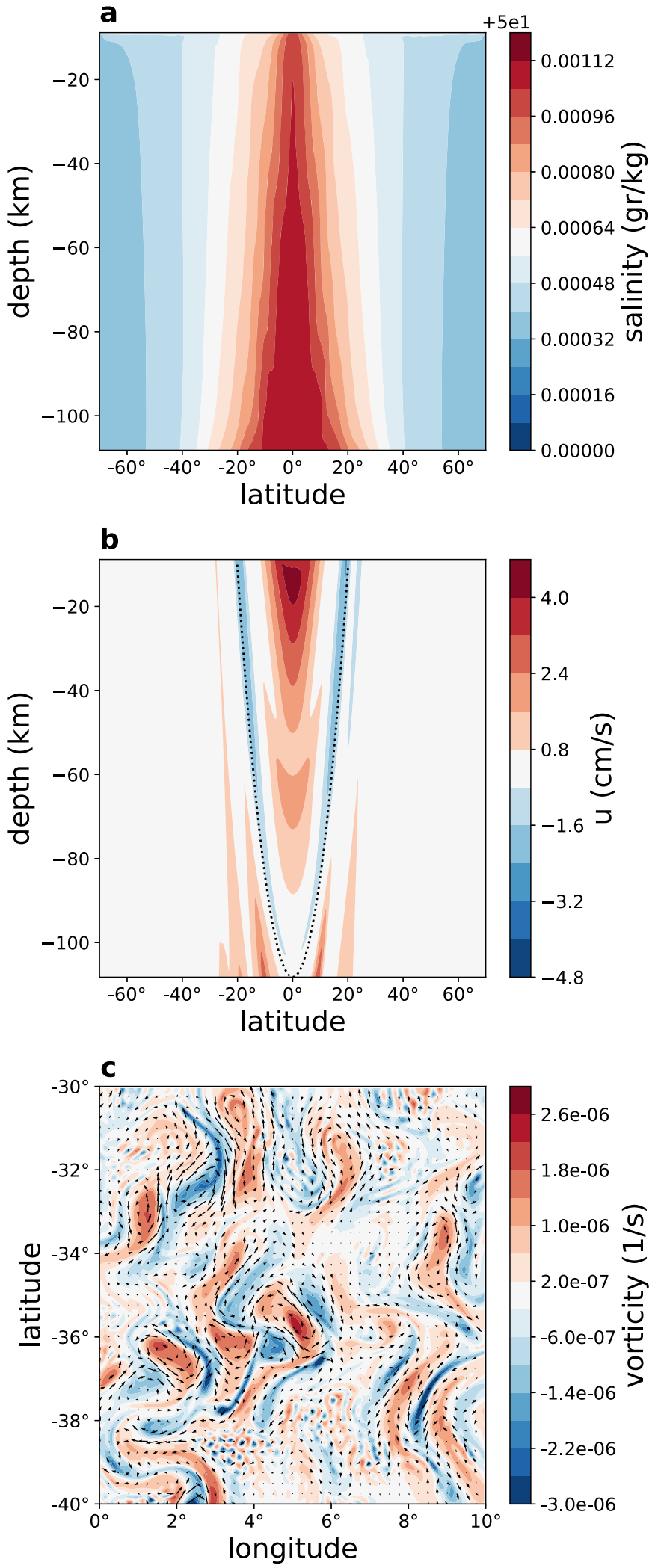

Fig. 5 Results of the 3d simulation-salinity, zonal velocity, and vorticity. a, b Latitude-depth plots of zonal mean salinity $(\mathrm{g} / \mathrm{kg})$ and zonal velocity $\left(\mathrm{cm} \mathrm{s}^{-1}\right) . \mathbf{c}$ Vorticity $\left(\mathrm{s}^{-1}\right)$ and velocity field (arrows) at the ocean surface, as a function of longitude and latitude, demonstrating the rotational fluid velocity around the Taylor columns.
References ${ }^{20,37}$ suggested the possibility of double diffusion in Europa's ocean. We find in the low latitudes in the $3 \mathrm{~d}$ model and in the high latitudes of the $2 \mathrm{~d}$ model, warm salty water under a surface layer of 1-2 grid points that is cold and fresh, and where the stratification is stable. While this is a scenario that can, in principle, lead to double diffusion and therefore to an enhanced vertical mixing, the surface layer is hardly resolved numerically and our results therefore do not seem to provide definite evidence for or against the idea that double diffusion may play a role in Europa's ocean.

One can get further insight into the eddy field from an energetic point of view. The oceanic available potential energy (APE) is the potential energy that may be converted into kinetic energy (KE). The ratio between the APE and the KE provides a measure of the efficiency of kinetic energy extraction from the stratification, and an indication of the source of eddy kinetic energy. For present-day Earth, the ratio between the APE and the $\mathrm{KE}$ ocean is over $33,000^{38}$. Europa's APE calculated following ${ }^{39}$ is $2.3 \times 10^{18} \mathrm{~J}$ for our $3 \mathrm{~d}$ model domain, only 190 times larger than the KE, a factor similar to that of the Snowball Earth ocean, where $\mathrm{APE} / \mathrm{KE}$ was estimated at about $300^{36}$. While the estimate of ${ }^{38}$ may be sensitive to the mean vertical density gradient used, we use the method of ref. ${ }^{39}$ because it does not depend on this gradient and is more appropriate for a very weakly stratified ocean. The ratio being much smaller in these Europa simulations is therefore a robust result. On Earth, macro-turbulence in the ocean and atmosphere is generated mostly by the drawing down of APE via baroclinic instability. Our results suggest that in Europa's ocean, convective plumes and barotropic instability of the zonal jets play a more prominent role in the generation of ocean macro-turbulence relative to baroclinic instability.

A previous study of the dynamics of the icy shell ${ }^{31}$ showed that an efficient meridional ocean heat transport can lead to a uniform shell thickness. The geothermal heat flux entering the ocean from below is larger than the heat escaping through the ice in the tropics and smaller at high latitudes, due to the meridional ice surface temperature gradient ${ }^{33,34}$. This would lead to melting at low latitudes and freezing at high latitudes, leading to ice thickness gradients balanced by ice flow ${ }^{31}$. However, an efficient poleward ocean heat transport can carry the excess heat meridionally, and thus overcome the tendency toward meridional ice thickness gradients, and result in almost uniform ice thickness (Fig. 6b). The meridional heat transports of the $2 \mathrm{~d}$ and $3 \mathrm{~d}$ ocean simulations are shown by the solid lines in Fig. 6 to be poleward, and have maximum values of about $0.5 \times 10^{11} \mathrm{~W}$ and $1.5 \times 10^{11} \mathrm{~W}$, correspondingly. These estimates of the meridional heat fluxes in a full ocean model are at least four times larger than the heat transport estimated by ref. ${ }^{31}$, because they include the contribution due to latent heat of freezing at the equator and melting at the poles, not included in previous studies.

The meridional heat flux without the latent heat contribution is shown by the green solid curve. This heat flux is determined, as explained above, by the geothermal and surface heat fluxes calculated for the assumed uniform thickness ice shell. The ocean has no difficulty transporting this heat flux in a way that is consistent with the uniform ice shell assumption. An ocean without an efficient meridional heat flux mechanism would have been heated in the tropics and cooled in the high latitude, not being able to reach a steady state. We conclude that the efficient ocean heat transport in our simulation is self-consistent with the assumption of a uniform ice thickness, justifying the use of a uniform thickness icy shell in this study. That the $3 \mathrm{~d}$ meridional heat flux is somewhat larger than the $2 \mathrm{~d}$ flux is a direct result of 

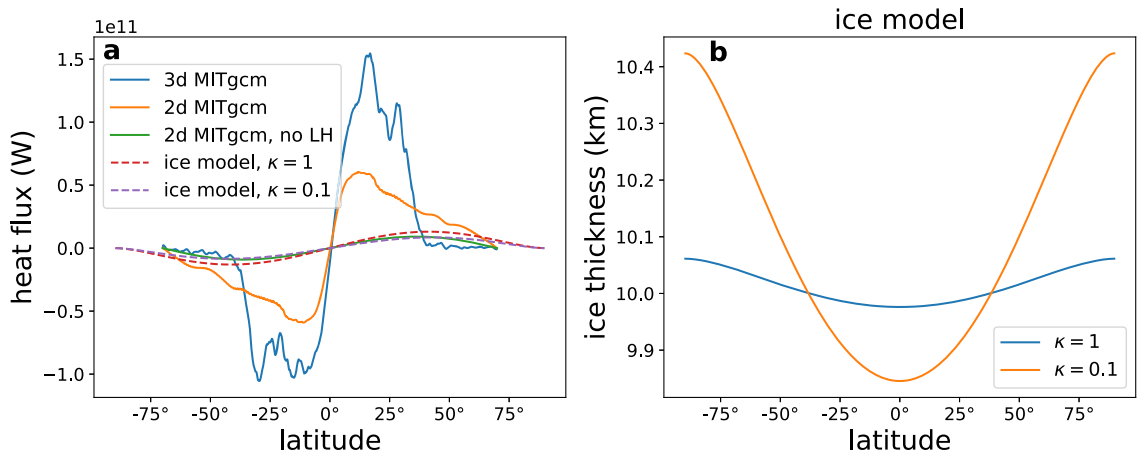

Fig. 6 Meridional ocean heat transport and implications for ice thickness. a The oceanic meridional heat transport calculated using the $2 \mathrm{~d}$ and $3 \mathrm{~d}$ ocean simulations in this work (solid lines). The heat transport is positive northward, so that the heat transport is poleward in both hemispheres. The green solid line shows the meridional transport calculated from the difference between the geothermal heat flux and the diffusive heat flux through the ice (text). Also shown (thin dash lines) are estimates of ocean heat transport using the slab ocean model of ref. ${ }^{31}$, coupled to an ice flow model, for two oceanic eddy mixing values, $\kappa=1,0.1 \mathrm{~m}^{2} \mathrm{~s}^{-1}$. b Ice thickness $(\mathrm{km})$ associated with the heat transport curves shown in a (dashed lines) showing almost uniform ice thickness even for relatively small eddy mixing coefficient of $\kappa=1 \mathrm{~m}^{2} \mathrm{~s}^{-1}$.

the larger latent heat due to freezing in the low latitudes in the $3 \mathrm{~d}$ case. The difference between the two model configurations is not large, and is within the uncertainty of the internal ocean variability, as estimated for example via the difference between the two hemispheres in the $3 \mathrm{~d}$ case. Spatial variations in tidal heating within the ice may still cause a range of surface heat fluxes and therefore ice thickness variations ${ }^{33,40}$, if the ice is sufficiently thick (thicker than chosen here based on ref. ${ }^{41}$ ) to allow convection.

\section{Discussion}

The $2 \mathrm{~d}$ and $3 \mathrm{~d}$ high resolution simulations of Europa's ocean analyzed here show a number of surprising results. While both the temperature and salinity are nearly uniform, salinity gradients, not considered previously, dominate the gradients in ocean water density, and the heaviest water (cold and saline) is found, as a result, at low latitudes. We showed this to be a result of the lack of direct communication along Taylor columns between the ocean bottom and surface in the area outside of the tangent cylinder. Taylor columns that are parallel to the axis of rotation are prevalent and show two regimes ${ }^{21}$, the low latitudes (outside of the tangent cylinder, equatorward of $\sim 20^{\circ}$ ) at which the Taylor columns do not intersect the ocean bottom and extend from one hemisphere to the other, and higher latitudes at which they intersect the ocean bottom. The Taylor columns, which were previously expected not to propagate in latitude due to potential vorticity conservation, and not to occupy the low-latitude regime $^{20}$, exhibit meridional propagation due to frictional effects and occupy the entire ocean. Their spacing was explained above in terms of the rotation rate and viscosity. The $3 \mathrm{~d}$ simulation shows a rich turbulent eddy flow, as well as convective plumes due to freezing and brine rejection near the ice-ocean interface, and due to geothermal heating from below. The convection plumes are perpendicular to the Taylor columns at low latitudes. We found superrotation at the equator, attributed it to eddy fluxes of zonal momentum and thermal wind balance, and attempted to explain the reasons for the difference in its structure between the $2 \mathrm{~d}$ and $3 \mathrm{~d}$ simulations. The meridional heat flux deduced here is much larger than previously estimated ${ }^{31}$, due to the contribution of the latent heat of freezing that was not considered in previous studies. The ratio between the APE and the $\mathrm{KE}$ is significantly smaller than on present-day Earth, yet similar to that estimated for the Snowball Earth ocean ${ }^{35,36,42}$.

A few recently submitted manuscripts investigate complementary aspects of the role of salinity in icy satellites to those discussed here, although they do not deal with the eddy motions and Taylor columns analyzed here. Reference ${ }^{43}$ examines the effect of ocean salinity on ice thickness and meridional ocean circulation. Reference ${ }^{44}$ examines the effects of low vs. high salinity on the circulation and stratification of Enceladus via the suppression of the water density anomaly by ocean salinity (see also our sensitivity experiments, Supplementary Figs. 1-5). Reference ${ }^{45}$ finds meridional overturning circulation and shallow freshwater polar lenses in Enceladus simulations.

Several of our above predictions may be verified in future missions to Europa, such as the Europa Clipper of NASA 46,47 or JUICE of ESA ${ }^{48}$. These include the uniform icy shell thickness due to the efficient meridional heat transport of Europa's ocean predicted in our simulations. The small meridionally variations of salinity predicted here may similarly be observable in future missions as well through its magnetic signal ${ }^{49}$ although this may be challenging. In addition to these observable predictions, the eddy diffusivity and viscosity coefficients-estimated here from an eddy-resolving simulation of Europa's ocean-should help in estimating ocean heat generation due to tides ${ }^{41}$. Similarly, the weak or even weakly unstable stratification suggests that internal wave breaking may not be a significant factor in tidal dissipation, consistent with previous estimates ${ }^{41}$. The libration of the icy shell may be influenced by ocean eddies and ocean currents and this may serve as a way of indirectly observing ocean dynamics. Finally, the study of Europa's ocean may help to better understand the ocean and ice dynamics of other icy moons/planets in the solar system and beyond.

\section{Methods}

Model description and configuration. To investigate the dynamics of Europa's ocean, we used the state-of-the-art Massachusetts Institute of Technology ocean GCM $\left(\mathrm{MITgcm}^{28,29}\right)$. This model configuration used here employs the fullynonlinear momentum equations for the ocean in height $(z)$-spherical coordinates, including the curvature terms, with a free surface. We use the non-hydrostatic option (rather than the more commonly used primitive equations which replace the vertical momentum equation with the hydrostatic approximation). The model simulates temperature and salinity using advection-diffusion equations, and uses a fully nonlinear equation of state relating them to the density field. The nonhydrostatic version of the MITgcm permits the use of the full Coriolis force, including the terms proportional to $2 \Omega \cos \phi$ that are often neglected when the aspect ratio (depth over horizontal scale) is small. Europa's aspect ratio is relatively large, and this option is therefore very important for Europa's ocean, as was anticipated by 20 .

The MITgcm was also used to study diverse oceanic phenomena, as well as the dynamics of other planets and moons, including Jupiter ${ }^{50,51}$, Pluto ${ }^{52,53}$, local convection on Europa ${ }^{22}$, Triton ${ }^{53}$, and hot Jupiter planets ${ }^{54,55}$. The model was used in the past to investigate the Snowball Earth events ${ }^{35,36,42,56}$, which share many similarities with Europa's ice-covered ocean. 
$2 \mathbf{d}$ and $3 \mathbf{d}$ configurations. We used two configurations, $2 \mathrm{~d}$ and $3 \mathrm{~d}$, correspondingly. Both configurations extend almost globally in the meridional direction $\left(70^{\circ} \mathrm{S}\right.$ to $70^{\circ} \mathrm{N}$ ). The depth of the ocean is set to $100 \mathrm{~km}$. The lateral resolution of the $2 \mathrm{~d}$ configuration is $1 / 12$ of a degree (about $2.3 \mathrm{~km}$ in the meridional direction) and we use 50 vertical levels with a $2 \mathrm{~km}$ uniform resolution. The $3 \mathrm{~d}$ simulation spans $30^{\circ}$ in the zonal direction with $1 / 24$ of a degree resolution, using 100 vertical levels with varying vertical resolution, ranging from $25 \mathrm{~m}$ at the top of the ocean to $1164 \mathrm{~m}$ at the bottom. These horizontal and vertical $3 \mathrm{~d}$ resolutions were found sufficient to resolve ocean eddy dynamics and convection processes. No-slip boundary conditions were assigned at the bottom and top (interface with the ice) of the ocean. The integration time steps were $7200 \mathrm{~s}$ and $400 \mathrm{~s}$ for the $2 \mathrm{~d}$ and $3 \mathrm{~d}$ configurations, respectively. Both simulations were ran for a sufficiently long time until statistical steady state was achieved.

Eddy coefficients, subgrid-scale representation. The model uses explicit eddy diffusion and viscosity coefficients that represent subgrid-scale mixing processes not resolved by the simulation. These are different from the eddy coefficients calculated below (Supplementary Fig. 12) which quantify the resolved eddy motions. The vertical explicit eddy viscosity and diffusion coefficients were set to $10^{-3}$ and $10^{-4} \mathrm{~m}^{2} \mathrm{~s}^{-1}$, respectively. The horizontal explicit eddy viscosity and tracer (thermal and haline) diffusion coefficients representing subgrid scale turbulent mixing are set to 50 and $5 \mathrm{~m}^{2} \mathrm{~s}^{-1}$, respectively, for the $2 \mathrm{~d}$ configuration and 20 and $2 \mathrm{~m}^{2} \mathrm{~s}^{-1}$ respectively for the $3 \mathrm{~d}$ configuration. The viscosity and diffusion coefficients are much larger than the molecular ones and are chosen to guarantee numerical stability. The eddy viscosity and diffusivity have different values, again a common practice in ocean modeling that is meant to allow using the smallest coefficients that lead to numerically stable results. The horizontal explicit eddy mixing coefficients are larger than the vertical ones, reflecting the different time scale of the subgrid scale turbulence represented by each.

These turbulent eddy coefficients can be formulated to be a function of the larger scale stratification and shear, as represented in present-day ocean model by the KPP parameterization, for example ${ }^{57}$. However, Europa's ocean is too different from Earth's to allow us to use such a parameterization, and we therefore set these coefficients to constant values. In addition, our non-hydrostatic simulations resolve the vertical convection plumes explicitly, even if marginally. We also do not use the Gent-McWilliams ${ }^{58}$ eddy parameterization that is commonly used in Earth's ocean simulations, because the large-slope isopycnals that develop in the simulations violate the assumptions used to derive this parameterization.

Ice shell and bottom boundary condition. Europa's icy shell is represented using the $\mathrm{MITg} \mathrm{cm}$ shelf-ice package ${ }^{29,30}$ that enables the calculation of the ocean-ice freshwater and heat fluxes based on the surface ice temperature, ice thickness, and ocean temperature and salinity ${ }^{30}$. The forcing ice surface temperature was taken from ref. ${ }^{34}$. Ice flow and dynamical ice thickness are not included in the shelf-ice package, but, as demonstrated above based on ref. ${ }^{31}$, the ice thickness can be assumed uniform due to the efficient meridional heat flux of the ocean (Fig. 6). A geothermal heating rate of $0.0496 \mathrm{~W} \mathrm{~m}^{-2}$ is specified at the ocean bottom; the exact bottom heating is not known (estimated to be between $5-200 \mathrm{~mW} \mathrm{~m}^{-2}$ ) and we use an intermediate estimate ${ }^{8,41,59}$ and performed sensitivity experiments to this value as shown in Supplementary Figs. 1-5. The internal heating leading to the geothermal heat flux is due to several sources: radiogenic heating of the metallic core and silicate mantle estimated at about $6-8 \mathrm{~mW} \mathrm{~m}^{-260}$, and tidal heating of Europa's core ${ }^{59}$. Tidal heating of the icy shell is not included explicitly, and tidal heating dissipation in the ocean is believed to be negligible ${ }^{41}$.

3-equation top boundary condition formulation. We use the 3 equationsformulation of ${ }^{29,30}$ to calculate the freshwater and heat fluxes between the ice shell and the ocean. The formulation represents an unresolved boundary layer just under the ice where these exchanges occur. According to these equations, the heat balance of the boundary layer is,

$$
c_{\mathrm{p}} \rho \gamma_{\mathrm{T}}\left(T-T_{\mathrm{f}}\right)+L q=\rho_{\mathrm{I}} c_{\mathrm{p}, \mathrm{I}} \kappa \frac{T_{\mathrm{f}}-T_{\mathrm{s}}}{h},
$$

where the $c_{\mathrm{p}}, c_{\mathrm{p}, \mathrm{I}}$ are the specific heat and water and ice, $\rho, \rho_{\mathrm{I}}$ are the density of ice and ocean, $\gamma_{\mathrm{T}}$ is the turbulent exchange coefficient of temperature, $T, T_{\mathrm{s}}$ is the top of the ocean and surface of the ice temperatures, $\kappa$ is the diffusion constant through the ice, $h$ is the thickness of the ice, and $L$ the latent heat constant of fusion. The freezing temperature, $T_{\mathrm{f}}$, depends both on the pressure at the bottom of the ice (which is uniform in our case as the ice thickness is uniform) and on the boundary layer salinity, $S_{\mathrm{b}}$, which is not uniform and calculated as part of the solution to the 3 -equation model. As a result, even when the ice thickness and the freezing temperature are constant (or almost constant), the heat flux into the ice which appears in these equations as the above $\rho_{\mathrm{I}} c_{\mathrm{p}, \mathrm{I}} \kappa \frac{T_{\mathrm{s}}-T_{\mathrm{f}}}{h}$ term, is not uniform in latitude as the temperature of the ice surface, $T_{\mathrm{s}}$, strongly varies by tens of degrees with latitude.

The upper boundary conditions for the temperature and salinity, that are behind the three-equation model used there, are essentially flux boundary conditions that take into account the effects of melting/freezing on both the salt concentration and heat fluxes due to freezing/melting and heat diffusion through the ice. The bottom boundary condition is again a flux boundary condition for both, specifying the geothermal heat flux and a no diffusive bottom flux for salt.

Differences in modeling strategy from previous studies of Europa's ocean Previous pioneering studies modeling Europa's ocean ${ }^{23,25}$ were based on the MagIC model used for core magnetohydrodynamics applications ${ }^{61}$, and is therefore different from the currently used ocean model which has been developed to study ocean dynamics in particular. Among the differences: the MagIC model is pseudo spectral, while the MITgcm used here is finite volume, they use isotropic viscosity/diffusivity, while modern ocean studies use non-isotropic coefficients (different in the vertical and horizontal directions in spherical coordinates, representing different expected efficiency of mixing in the two directions). As mentioned in the paper body, the top and bottom boundary conditions are of prescribed temperature in the above studies, while we use a more self-consistent formulation involved a flux condition at the bottom and a 3 -equation model at the bottom of the ice shelf. We explicitly represent the ice shelf, its melting, freezing and heat conduction-all of which were ignored previously. These previous studies ignored salinity effects which are included here, and these salinity effects are found to dominate the density distribution as explained in the article. The previous studies used a linear equation of state relating the density to the temperature, while we use a full-complexity nonlinear equation of state relating density to temperature, salinity and pressure ${ }^{62}$. Finally, our resolution in both $2 \mathrm{~d}$ and $3 \mathrm{~d}$ is significantly higher than previously used.

Sensitivity to mean salinity, bottom heating, and ice thickness. Estimates of Europa's ocean salinity vary widely, from the ocean being nearly fresh to highly saline ${ }^{63}$. Importantly, the magnetometer on the Europa Clipper may be able to estimate the mean ocean salinity ${ }^{46,47}$. The mean salinity affects the freezing temperature of ice and the ocean dynamics, as density variations are found in this work to be driven mostly by salinity gradients rather than temperature gradients. We have used a moderate salinity value of $50 \mathrm{~g} / \mathrm{kg}(\mathrm{ppt})$ as our default value. Given the uncertainty in this mean salinity value, we summarize in Supplementary Figs. 1-5 a set of $2 \mathrm{~d}$ sensitivity tests that include mean salinity of $10^{-6} \mathrm{ppt}, 5 \mathrm{ppt}, 10 \mathrm{ppt}$, and $25 \mathrm{ppt}$. We also test the sensitivity of our results to the ice thickness, noting that the ice thickness should be in equilibrium with the geothermal bottom heat flux: as the thickness is changed, the diffusive heat flux through the ice changes, and a steady state requires the globally integrated heat flux through the ice to be equal to the integrated bottom heat flux. We used ice thickness values of $5 \mathrm{~km}$ (which is in equilibrium with an ocean bottom heat flux of $100 \mathrm{~mW} \mathrm{~m}^{-2}$ ) and $15 \mathrm{~km}$ (corresponding to ocean bottom heat flux of $33 \mathrm{~mW} \mathrm{~m}^{-2}$ ).

In all the simulations, we find that the coldest water is in the upper ocean, outside the tangent cylinder, as in our default simulation analyzed in the paper itself (Supplementary Figs. 1-5). The salinity is maximal outside the tangent cylinder (around the equator), except for the freshwater case (Supplementary Fig. 2a). The density is maximal outside the tangent cylinder for all simulation except the two lowest mean salinities (Supplementary Fig. 3a, b) for which the anomaly of sea water leads to denser water at the bottom due to the bottom heating there. The top to bottom temperature difference is robustly at around $0.01^{\circ} \mathrm{C}$, even when the bottom heat flux is changed from our default value. In all simulations the flow is westward except the bottom equatorial region for which superrotation is observed as discussed in the paper. The Taylor columns structure is clearly visible in the meridional velocity and is similar in all simulations, consistent with the results presented in the main text. Moreover, the structure of the temperature, salinity, and density fields is similar to the structure of those presented in the main text (Fig. 1). We conclude that the sensitivity simulations indicate the robustness of the results of the default experiment analyzed in the paper.

The dominant ocean momentum balance. In order to explore the momentum balance of Europa's ocean, we use the output of the model that uses the full set of equations as explained above, but consider only those terms that are not negligible. We therefore consider the following equations, assuming zonal symmetry, and neglecting the small curvature terms. The momentum equations are then

$$
\begin{array}{r}
u_{\mathrm{t}}+\frac{1}{a} v u_{\phi}+w u_{\mathrm{z}}-2 \Omega \sin (\phi) v+2 \Omega \cos (\phi) w=\frac{\nu_{\mathrm{h}}}{a^{2} \cos (\phi)}\left(\cos (\phi) u_{\phi}\right)_{\phi}+\nu_{\mathrm{v}} u_{z z} \\
v_{\mathrm{t}}+\frac{1}{a} v v_{\phi}+w v_{\mathrm{z}}+2 \Omega \sin (\phi) u=-\frac{1}{a \rho_{0}} p_{\phi}+\frac{\nu_{\mathrm{h}}}{a^{2} \cos (\phi)}\left(\cos (\phi) v_{\phi}\right)_{\phi}+\nu_{\mathrm{v}} v_{z z} \\
w_{\mathrm{t}}+\frac{1}{a} v w_{\phi}+w w_{\mathrm{z}}-2 \Omega \cos (\phi) u=-\frac{1}{\rho_{0}} p_{\mathrm{nhz}}+\frac{\nu_{\mathrm{h}}}{a^{2} \cos (\phi)}\left(\cos (\phi) w_{\phi}\right)_{\phi}+\nu_{\mathrm{v}} w_{z z} \\
p_{\mathrm{hdz}}=-g \rho \\
p=p_{\mathrm{hd}}+p_{\mathrm{nh}},
\end{array}
$$

where $\phi, z, t$ indicate the latitude, depth, and time, $u, v, w$ are the zonal, meridional, and vertical velocities, $p_{\text {hd,nh }}$ is the hydrostatic/nonhydrostatic pressure, $\rho$ is the density, $a$ is the radius of Europa, $\Omega$ is the rotation frequency, $\nu_{h, v}$ are horizontal and vertical viscosity coefficients, and $g$ is the gravity acceleration. The continuity equation assuming again zonal symmetry is,

$$
\frac{1}{a \cos (\phi)}(v \cos (\phi))_{\phi}+w_{z}=0 .
$$


The different terms in the momentum and continuity equations are shown in Supplementary Figs. 14-16. The Coriolis terms dominate the zonal horizontal momentum equation, followed by the horizontal viscosity term. In the meridional momentum equation, the balance is geostrophic: the horizontal Coriolis term balances the pressure gradient term, and the horizontal viscosity term is smaller yet not completely negligible.

While the model simulations shown in this work use a fully nonlinear equation of state ${ }^{62}$ relating density to temperature, salinity and pressure, we note that a linearized equation can be written as $\rho=\rho_{0}\left(1-\alpha\left(T-T_{0}\right)+\beta\left(S-S_{0}\right)\right)$ where $\alpha, \beta$ are the expansion coefficients mentioned in the main text. Because the temperature and salinity variations are very small, this linearized approximation is very accurate.

We now wish to explain the meridional symmetry of the velocity parallel to the axis of rotation, and the meridional anti-symmetry of the velocity perpendicular to the axis of rotation, as seen in Fig. 1b, c. The dominant terms in the zonal momentum equation are the Coriolis terms (Supplementary Fig. 15a, b) and the balance between them yields,

$$
w \cos (\phi) \approx v \sin (\phi) .
$$

This is more easily understood by writing the geostrophic approximation in cylindrical coordinates,

$$
2 \Omega v_{\mathrm{r}} \approx-\frac{1}{r \rho} \frac{\partial p}{\partial \theta},
$$

where now $v_{\mathrm{r}}$ is the velocity perpendicular to the axis of rotation, written in terms of the spherical coordinate velocity field as $v_{\mathrm{r}}=w \cos \phi-v \sin \phi$ and $\theta$ is the longitude. The $2 \mathrm{~d}$ model configuration assumes zonal symmetry (no variations in $\theta$ ), so that the last equation implies $v_{r} \approx 0$, exactly equivalent to (4) in spherical coordinates.

The simple relation (4), written as $w \approx v \tan (\phi)$, does not depend on any parameters and reflects the symmetry properties of $v, w$ : The symmetry of $w$ is opposite of the symmetry of $v \operatorname{since} \tan (\phi)$ is anti-symmetric about the equator. Finally, the velocity parallel to the axis of rotation can be expressed in terms of the meridional and vertical velocities $v, w$ as $v_{\mathrm{z}}=w \sin \phi+v \cos \phi$ and since $w \cos \phi \approx v \sin \phi, v_{z}=v / \cos \phi$, implying that $v_{z}$ is symmetric as $v$.

The spacing between the Taylor columns. Based on Supplementary Figs. 14-16, the dominant terms in the zonal and meridional momentum equations near the top of the ocean, where we find viscosity to be non-negligible (2) are,

$$
\begin{aligned}
-2 \Omega \sin (\phi) v & =\frac{\nu_{\mathrm{h}}}{a^{2} \cos (\phi)}\left(\cos (\phi) u_{\phi}\right)_{\phi} \\
2 \Omega \sin (\phi) u & =-\frac{1}{a \rho_{0}} p_{\phi}+\frac{\nu_{\mathrm{h}}}{a^{2} \cos (\phi)}\left(\cos (\phi) v_{\phi}\right)_{\phi}
\end{aligned}
$$

The term $2 \Omega \cos (\phi) w$ in the first equation is small in the upper $10 \mathrm{~km}$ or so of the ocean due to the no-normal flow conditions (Supplementary Fig. 15b). The viscosity term is generally smaller than the Coriolis term, especially in the interior (Supplementary Fig. 16a, b) in which geostrophy holds, $2 \Omega \sin (\phi) \bar{u}=-\frac{1}{a \rho_{0}} p_{\phi}$, where $\bar{u}$ denotes the geostrophic zonal velocity. Then, the meridional momentum of Eqs. (6) can be written as,

$$
2 \Omega \sin (\phi)(u-\bar{u})=\frac{\nu_{\mathrm{h}}}{a^{2} \cos (\phi)}\left(\cos (\phi) v_{\phi}\right)_{\phi} .
$$

Assuming that the meridional gradient of the geostrophic term is small, we can approximate $u_{\phi} \approx(u-\bar{u})_{\phi}$. This heuristic argument is supported by the smoother interior structure of the zonal velocity seen in Fig. 2a. Eq. (6) can now be written as,

$$
\begin{aligned}
-2 \Omega \sin (\phi) v & =\frac{\nu_{h}}{a^{2} \cos (\phi)}\left(\cos (\phi) \tilde{u}_{\phi}\right)_{\phi} \\
2 \Omega \sin (\phi) \tilde{u} & =\frac{\nu_{h}}{a^{2} \cos (\phi)}\left(\cos (\phi) v_{\phi}\right)_{\phi},
\end{aligned}
$$

where $\tilde{u}=u-\bar{u}$. Using a complex variable $\alpha=\tilde{u}+i v$ the above equations can be written in terms of a single differential equation that holds near the top of the ocean where viscosity is non-negligible,

$$
i 2 \Omega \sin (\phi) \alpha=\frac{\nu_{\mathrm{h}}}{a^{2} \cos (\phi)}\left(\cos (\phi) \alpha_{\phi}\right)_{\phi} \approx \frac{\nu_{h}}{a^{2}} \alpha_{\phi \phi},
$$

or

$$
\alpha_{\phi \phi}-i k_{\phi}^{2} \alpha=0 ; k_{\phi}^{2}=\frac{2 \Omega a^{2}}{\nu_{\mathrm{h}}} \sin (\phi) .
$$

Given that the wave number $k_{\phi}^{2}$ is a slowly varying function of latitude (relative to the meridional scale of the Taylor columns), it can now be used to estimate the spacing between the Taylor columns. The corresponding wavelength in spherical coordinates is $\lambda=2 \pi / k_{\phi}$. The distance of a given point, at a latitude $\phi$, along the ocean surface from the axis of rotation is given by $a \cos \phi$. The distance between two adjacent columns in the direction perpendicular to the axis of rotation, is therefore,

$$
d(\phi)=a[\cos (\phi-\lambda / 2)-\cos (\phi+\lambda / 2)]=2 a \sin (\phi) \sin (\lambda / 2) \approx a \lambda \sin (\phi) .
$$

Or, more explicitly

$$
d(\phi)=\sqrt{2} \pi \sqrt{\frac{\nu_{h}}{\Omega} \sin (\phi)} .
$$

This approximation reproduces the functional behavior shown in Fig. 3a, although a factor that seems to be about $\pi$ is missing to be consistent with the numerical fit to the simulated distances. The above arguments are admittedly heuristic at best, yet suggest that eddy viscosity may indeed be at the heart of the process that sets the Taylor column spacing.

Following ref. ${ }^{20}$, we considered explaining the spacing between the Taylor columns through the Rhines scale, $L_{\rho}=\sqrt{2 U / \beta}$. However, this scale does not seem to match the simulated spacing (more clearly shown by $u_{\text {par }}, u_{\text {per, }}$ Fig. $2 \mathrm{~b}, \mathrm{c}, \mathrm{e}$ ). At the equator $\beta=2.6 \times 10^{-11} \mathrm{~m}^{-1} \mathrm{~s}^{-1}$ and for a typical velocity of $U \sim 0.02 \mathrm{~m} \mathrm{~s}^{-1}$ the Rhines scale is $L_{\rho} \approx 40 \mathrm{~km}$, larger than the Taylor column spacing at the equator (between 10 and $20 \mathrm{~km}$ ). For larger latitudes the Rhines scale becomes larger due to the division by $\beta$ which is proportional to the cosine of latitude. In contrast, the spacing we observe seems proportional to $\sqrt{\sin \phi}$ ( $\phi$ is the latitude, Fig. 3), consistent with our revised explanation, and not to $\sqrt{1 / \cos \phi}$ as predicted by the Rhines scale.

The scaling of ref. ${ }^{64}$ (their equation 23 , based on their equation 22) in the case of rotating planet relates the Taylor column height $(D)$ to its horizontal scale $(L)$ as $L / D \sim\left(\frac{\nu_{\mathrm{h}}}{2 \Omega D^{2}}\right)^{1 / 3}$ so that $L \sim\left(\frac{D \nu_{\mathrm{h}}}{2 \Omega}\right)^{1 / 3}$. One can see that this cannot apply in our case for two reasons. First, there is a jump by a factor of two in the Taylor column height in our case across the tangent cylinder, but no jump is seen in the Taylor column spacing. Second, the Taylor column height in our Europa simulations first increases with latitude from the equator to the tangent cylinder and then decreases with latitude for higher latitudes. Their scaling would predict that the horizontal scale is therefore not monotonous in latitude (i.e., the spacing between the Taylor columns increases from the equator towards the tangent cylinder and then decreases towards the higher latitudes), in contrast to our numerical finding (Fig. 3) and to our own scaling arguments (given above) of monotonically increasing Taylor spacing from the equator towards the high latitudes.

Estimating eddy coefficients. We estimate an horizontal eddy mixing coefficient, $\kappa_{\mathrm{h}}$, that effectively represents the effects of ocean macro-turbulence, using time series of the zonal velocity at multiple grid points ${ }^{65,66}$. We use the auto-correlation function, $R(\tau)$, and the variance of the zonal velocity temporal anomaly as follows: $\kappa_{\mathrm{h}}=\overline{u_{\mathrm{L}}^{\prime 2}} \int_{0}^{\infty} R(\tau) \mathrm{d} \tau$, where $u_{\mathrm{L}}$ is the Lagrangian zonal velocity, the overbar indicates mean over time while the prime indicates the temporal anomaly around this mean. When using the Eulerian velocity field, it is necessary to multiply $\kappa_{\mathrm{h}}$ by a constant $\gamma$ which we choose to be $\gamma=4^{36,66}$. The estimated eddy parameterized viscosity coefficient is larger or equal to the estimated diffusion coefficient ${ }^{36,66}$

We find that the estimated diffusion coefficient depends on latitude, where for latitudes larger than $40^{\circ}$ the diffusion coefficient is smaller than $40 \mathrm{~m}^{2} \mathrm{~s}^{-1}$ while of latitudes smaller than $40^{\circ}$ it can reach a value larger than $1000 \mathrm{~m}^{2} \mathrm{~s}^{-1}$, depending on the latitude. The diffusion coefficient is also larger near the ocean bottom. We also estimated the diffusion/viscosity coefficient using an alternative approach based on the deviations of the zonal velocity from the zonal mean, and by estimating a characteristic length scale through the auto-correlation function in the zonal direction and multiplying it by the (zonal) standard deviation of the zonal velocity. We find diffusion/viscosity coefficients that are fairly similar to those found in the first method, of about $300 \mathrm{~m}^{2} \mathrm{~s}^{-1}$, again stronger at latitudes smaller than $40^{\circ}$ and largest near the ocean bottom. Thus, following the above, a rough lower bound for the global mean eddy mixing coefficient is about $200 \mathrm{~m}^{2} \mathrm{~s}^{-1}$. Since the viscosity coefficient is usually larger than the diffusion coefficient, the above estimate for the viscosity coefficient of $300 \mathrm{~m}^{2} \mathrm{~s}^{-1}$ seems reasonable.

Surprisingly, these values are only an order of magnitude smaller than those estimated for Earth's ocean (e.g., $\sim 1000-5000 \mathrm{~m}^{2} \mathrm{~s}^{-1}$ in the tropical ocean ${ }^{67}$ ) and of the same order as estimated for an Earth Snowball ocean ${ }^{36}$, indicating that in spite of the lack of wind forcing and a direct solar forcing of the ocean, ocean eddies can develop from internal instabilities and are playing a dominant role in Europa's ocean dynamics and heat transport.

Scaling estimates of the role of rotation in convection dynamics. Previous studies ${ }^{68}$ have suggested based on scaling arguments that rotation should affect the convection regime and therefore the top-to-bottom temperature difference. These scaling arguments assume a single component fluid (i.e., only temperature affecting the density), yet in our simulations the density variations are dominated by the salinity, while the temperature is close to the freezing temperature and therefore has only a small effect on the density. While the standard scaling is therefore not applicable in our case, we still calculate the modified and conventional nondimensional Rayleigh and Nusselt numbers based on the coefficients used in our Europa simulations and find that the conventional nondimensional numbers are much larger (by orders of magnitudes) than the modified numbers, indicating that rotation is not expected to play a role in the convection process. Specifically, the Prandtle number is, $\operatorname{Pr}=v_{\mathrm{v}} / \kappa_{\mathrm{v}}\left(\nu_{\mathrm{v}}\right.$ is the vertical viscosity coefficient and $\kappa_{\mathrm{v}}$ is the vertical diffusion coefficient). The Ekman number is $E=v_{\mathrm{v}} /\left(\Omega D^{2}\right)(\Omega$ is the rotation frequency and $D$ icy shell thickness). The thermal Ekman number is $\mathrm{Ek}=\kappa_{\mathrm{v}} /\left(\Omega D^{2}\right)=E / \mathrm{Pr}$. The modified Rayleigh number in the presence of rotation 
is $\mathrm{Ra}^{*}=(\alpha \Delta T g) /\left(\Omega^{2} D\right)$, while the conventional Rayleigh number is $\mathrm{Ra}=\mathrm{Ra}^{*}$ / (Ek E). Similarly, the conventional Nusselt number is $\mathrm{Nu}=q D /\left(\rho c_{\mathrm{p}} \kappa_{\mathrm{v}} \Delta T\right)$ where $q$ is the heat flux, while the modified Nusselt number in the presence of rotation is $\mathrm{Nu}^{*}=(\mathrm{Nu}-1) \mathrm{Ek}$. Calculating these nondimensional numbers for values corresponding to our Europa simulations, we find, $\Omega=2 \times 10^{-5} \mathrm{~s}^{-1}, \kappa_{\mathrm{v}}=1 \times 10^{-4} \mathrm{~m}^{2} \mathrm{~s}^{-1}$, $v_{\mathrm{v}}=1 \times 10^{-3} \mathrm{~m}^{2} \mathrm{~s}^{-1}, D=10^{5} \mathrm{~m}, \operatorname{Pr}=10, g=1.314 \mathrm{~m} \mathrm{~s}^{-2}, q=0.05 \mathrm{Wm}^{-2}$, $\alpha \Delta T=\Delta \rho / \rho=2 \times 10^{-6}, E=5 \times 10^{-9}, \mathrm{Ek}=5 \times 10^{-10}, \mathrm{Ra}^{*}=\left(2 \times 10^{-6}\right) /$ $\left(4 \times 10^{-10} \times 10^{5}\right)=0.05, \mathrm{Ra}=0.05 /\left(5 \times 10^{-10} \times 5 \times 10^{-9}\right)=2 \times 10^{16}, \mathrm{Nu}=0.05 \times 10^{5} /$ $\left(10^{3} \times 4 \times 10^{3} \times 10^{-4} \times 0.01\right)=1250, \mathrm{Nu}^{*}=(\mathrm{Nu}-1) \mathrm{Ek}=6 \times 10^{-7}$. Thus, the conventional Rayleigh and Nusselt numbers are much larger than the modified ones, indicating that the rotation is not expected to play a major role in the convection process. It is possible to define a Nusselt number that depends on density variations and not temperature. It is $\mathrm{Nu}=q /\left(g \kappa_{\mathrm{v}} \Delta \rho\right)$, which yields $\mathrm{Nu}=0.05 /$

$\left(10^{-4} \times 2 \times 10^{-4}\right)=2.5 \times 10^{6}$, so that again the rotation is not expected to be a major factor, even when taking salinity changes into account.

\section{Data availability}

The datasets generated during and/or analysed during the current study are available from the corresponding author on reasonable request. The model's setup files are available in the OSF repository, http://OSF.IO/SVXBQ, https://doi.org/10.17605/OSF.IO/ SVXBQ.

\section{Code availability}

The reported results were generated using the MITgcm code which can be downloaded from https://github.com/MITgcm/MITgcm or https://doi.org/10.5281/zenodo.1409237.

Received: 28 January 2021; Accepted: 19 October 2021;

Published online: 04 November 2021

\section{References}

1. Chyba, C. F. \& Phillips, C. B. Possible ecosystems and the search for life on Europa. Proc. Natl Acad. Sci. USA 98, 801-804 (2001).

2. Hand, K., Chyba, C., Priscu, J., Carlson, R. \& Nealson, K. In Astrobiology and the Potential for Life on Europa (eds Pappalardo, R. T. et al.) 589-629 (University of Arizona Press, 2009).

3. Pappalardo, R. et al. Science potential from a Europa lander. Astrobiology 13, 740-773 (2013).

4. Cassen, P., Reynolds, R. T. \& Peale, S. Is there liquid water on Europa? Geophys. Res. Lett. 6, 731-734 (1979)

5. Carr, M. H. et al. Evidence for a subsurface ocean on Europa. Nature 391 , 363-365 (1998).

6. Kivelson, M. G. et al. Galileo magnetometer measurements: a stronger case for a subsurface ocean at Europa. Science 289, 1340-1343 (2000).

7. Billings, S. E. \& Kattenhorn, S. A. The great thickness debate: Ice shell thickness models for Europa and comparisons with estimates based on flexure at ridges. Icarus 177, 397-412 (2005).

8. Hussmann, H., Spohn, T. \& Wieczerkowski, K. Thermal equilibrium states of Europa's ice shell: implications for internal ocean thickness and surface heat flow. Icarus 156, 143-151 (2002)

9. Tobie, G., Choblet, G. \& Sotin, C. Tidally heated convection: constraints on Europa's ice shell thickness. J. Geophys. Res. 108, 5124 (2003).

10. Bierhaus, E. B. et al. In Europa's Crater Distributions and Surface Ages (eds Pappalardo, R. T. et al.) 161-180 (University of Arizona Press, 2009).

11. Schmidt, B., Blankenship, D., Patterson, G. \& Schenk, P. Active formation of 'chaos terrain' over shallow subsurface water on Europa. Nature 479, 502-505 (2011).

12. Khurana, K. et al. Induced magnetic fields as evidence for subsurface oceans in Europa and Callisto. Nature 395, 777-780 (1998).

13. Pappalardo, R. et al. Does Europa have a subsurface ocean? Evaluation of the geological evidence. J. Geophys. Res. 104, 24015-24055 (1999).

14. Roth, L. et al. Transient water vapor at Europa's south pole. Science 343, 171-174 (2014).

15. Sparks, W. et al. Probing for evidence of plumes on Europa with HST/STIS. Astrophys. J. 829, 121 (2016).

16. Thomson, R. E. \& Delaney, J. R. Evidence for a weakly stratified Europan ocean sustained by seafloor heat flux. J. Geophys. Res. 106, 12355-12365 (2001).

17. Goodman, J. C., Collins, G. C., Marshall, J. \& Pierrehumbert, R. T. Hydrothermal plume dynamics on Europa: implications for chaos formation. J. Geophys. Res. 109, E03008 (2004).

18. Melosh, H., Ekholm, A., Showman, A. \& Lorenz, R. The temperature of Europa's subsurface water ocean. Icarus 168, 498-502 (2004).

19. Tyler, R. H. Strong ocean tidal flow and heating on moons of the outer planets. Nature 456, 770-772 (2008).
20. Vance, S. \& Goodman, J. In Oceanography of an Ice-covered Moon (eds Pappalardo, R. T. et al.) 459-482 (The University of Arizona Press, 2009).

21. Goodman, J. C. Tilted Geostrophic Convection in Icy World Oceans Caused by the Horizontal Component of the Planetary Rotation Vector (American Geophysical Union, Fall Meeting 2012, abstract, P51A-2017, 2012).

22. Goodman, J. C. \& Lenferink, E. Numerical simulations of marine hydrothermal plumes for Europa and other icy worlds. Icarus 221, 970-983 (2012).

23. Soderlund, K. M., Schmidt, B. E., Wicht, J. \& Blankenship, D. D. Ocean-driven heating of Europa's icy shell at low latitudes. Nat. Geosci. 7, 16-19 (2014).

24. Gissinger, C. \& Petitdemange, L. A magnetically driven equatorial jet in europa's ocean. Nat. Astron. 3, 401 (2019).

25. Soderlund, K. M. Ocean dynamics of outer solar system satellites. Geophys. Res. Lett. 46, 8700-8710 (2019).

26. Rovira-Navarro, M. et al. Do tidally-generated inertial waves heat the subsurface oceans of europa and enceladus? Icarus 321, 126-140 (2019).

27. Lemasquerier, D. et al. Libration-driven flows in ellipsoidal shells. J. Geophys Res. 122, 1926-1950 (2017).

28. Marshall, J., Adcroft, A., Hill, C., Perelman, L. \& Heisey, C. A finite-volume, incompressible Navier Stokes model for studies of the ocean on parallel computers. J. Geophys. Res. 102,C3, 5753-5766 (1997)

29. MITgcm Group. MITgcm User Manual. Online documentation. (MIT/EAPS, 2021). https://mitgcm.readthedocs.io/en/latest/.

30. Losch, M. Modeling ice shelf cavities in a $\mathrm{z}$-coordinate ocean general circulation model. J. Geophys. Res. 113, C08043 (2008).

31. Ashkenazy, Y., Sayag, R. \& Tziperman, E. Dynamics of the global meridional ice flow of Europa's icy shell. Nat. Astron. 2, 43 (2018).

32. Hand, K. P. \& Chyba, C. F. Empirical constraints on the salinity of the europan ocean and implications for a thin ice shell. Icarus 189, 424-438 (2007).

33. Ojakangas, G. W. \& Stevenson, D. J. Thermal state of an ice shell on Europa. Icarus 81, 220-241 (1989).

34. Ashkenazy, Y. The surface temperature of Europa. Heliyon. 5, e01908 (2019)

35. Jansen, M. F. The turbulent circulation of a Snowball Earth ocean. J. Phys. Oceanogr. 46, 1917-1933 (2016)

36. Ashkenazy, Y. \& Tziperman, E. Variability, instabilities and eddies in a Snowball ocean. J. Clim. 29, 869-888 (2016).

37. Vance, S. \& Brown, J. M. Layering and double-diffusion style convection in Europa's ocean. Icarus 177, 506-514 (2005)

38. vonStorch, J. S. et al. An estimate of the lorenz energy cycle for the world ocean based on the storm/ncep simulation. J. Phys. Oceanogr. 42, 2185-2205 (2012).

39. Huang, R. X. Mixing and energetics of the oceanic thermohaline circulation. J. Phys. Oceanogr. 29, 727-746 (1999).

40. Sotin, C., Tobie, G., Wahr, J. \& McKinnon, W. B. in Tides and Tidal Heating on Europa (eds Pappalardo, R. T. et al.) 85-117 (The University of Arizona Press, 2009).

41. Chen, E. M. A., Nimmo, F. \& Glatzmaier, G. A. Tidal heating in icy satellite oceans. Icarus 229, 11-30 (2014).

42. Ashkenazy, Y. et al. Dynamics of a snowball Earth ocean. Nature 495, 90-93 (2013).

43. Kang, W., Mittal, T., Bire, S., Michel, J. \& Marshall, J. How does salinity shape ocean circulation and ice geometry on enceladus and other icy satellites? https://arXiv.org/2104.07008 (2021)

44. Zeng, Y. \& Jansen, M. F. Ocean circulation on enceladus with a high- versus low-salinity ocean. Planet. Sci. J. 2, 151 (2021).

45. Lobo, A. H., Thompson, A. F., Vance, S. D. \& Tharimena, S. A pole-toequator ocean overturning circulation on enceladus. Nat. Geosci. 14, 185-189 (2020).

46. Pappalardo, R. et al. Science objectives and capabilities of the NASA Europa mission. In Lunar and Planetary Science Conference, vol. 47, 3058 (2016).

47. Howell, S. M. \& Pappalardo, R. T. Nasa's europa clipper-a mission to a potentially habitable ocean world. Nat. Commun. 11, 1-4 (2020).

48. Grasset, O. et al. JUpiter ICy moons Explorer (JUICE): An ESA mission to orbit Ganymede and to characterise the Jupiter system. Planet. Space Sci. 78, 1-21 (2013)

49. Vance, S. D. et al. Magnetic induction responses of Jupiter's ocean moons including effects from adiabatic convection. J. Geophys. Res. 126 e2020JE006418 (2021)

50. Kaspi, Y. Turbulent convection in an anelastic rotating sphere: a model for the circulation on the giant planets. Ph.D. thesis, (2008)

51. Kaspi, Y., Flierl, G. R. \& Showman, A. P. The deep wind structure of the giant planets: results from an anelastic general circulation model. Icarus 202, 525-542 (2009)

52. Zalucha, A. \& Gulbis, A. Comparison of a simple 2-D Pluto general circulation model with stellar occultation light curves and implications for atmospheric circulation. J. Geophys. Res. 117, E05002 (2012). 
53. Zalucha, A. M. \& Michaels, T. I. A 3D general circulation model for Pluto and Triton with fixed volatile abundance and simplified surface forcing. Icarus 223, 819-831 (2013)

54. Showman, A. P. et al. Atmospheric circulation of hot Jupiters: coupled radiative-dynamical general circulation model simulations of HD 189733b and HD 209458b. Astrophys. J. 699, 564 (2009).

55. Parmentier, V., Fortney, J. J., Showman, A. P., Morley, C. \& Marley, M. S. Transitions in the cloud composition of hot Jupiters. Astrophys. J. 828, 22 (2016).

56. Ashkenazy, Y., Gildor, H., Losch, M. \& Tziperman, E. Ocean circulation under globally glaciated Snowball Earth conditions: steady state solutions. J. Phys. Oceanogr. 44, 24-43 (2014).

57. Large, W. G., Mcwilliams, J. C. \& Doney, S. C. Oceanic vertical mixing: a review and a model with a nonlocal boundary-layer parameterization. Rev. Geophys. 32, 363-403 (1994).

58. Gent, P. R. \& McWilliams, J. C. Isopycnal mixing in ocean circulation models. J. Phys. Oceanogr. 20, 150-155 (1990).

59. Greenberg, R., Geissler, P., Hoppa, G. \& Tufts, B. Tidal-tectonic processes and their implications for the character of Europa's icy crust. Rev. Geophys. 40, 1-1 (2002).

60. Barr, A. C. \& Showman, A. P. in Heat Transfer in Europa's Icy Shell (eds Pappalardo, R. T. et al.) 405-430 (The University of Arizona Press, 2009).

61. Christensen, U. R. \& Wicht, J. Numerical dynamo simulations. Treatise Geophys. 8, 245-277 (2015).

62. Jackett, D. R. \& McDougall, T. J. Minimal adjustment of hydrographic profiles to achieve static stability. J. Atmos. Ocean. Technol. 12, 381-389 (1995).

63. Zolotov, M. Y. \& Kargel, J. In On the Chemical Composition of Europa's Icy Shell, Ocean, and Underlying Rocks (eds Pappalardo, R. T. et al.) 431-457 (University of Arizona Press, 2009).

64. Zhang, K. \& Schubert, G. Magnetohydrodynamics in rapidly rotating spherical systems. Annu. Rev. Fluid Mech. 32, 409-443 (2000).

65. Hinze, J. O. Turbulence (McGrow-Hill, 1975).

66. Lemmin, U. Dynamics of horizontal turbulent mixing in a nearshore zone of Lake Geneva. Limnol. Oceanogr. 34, 420-434 (1989).

67. Abernathey, R. P. \& Marshall, J. Global surface eddy diffusivities derived from satellite altimetry. J. Geophys. Res. 118, 901-916 (2013).

68. Christensen, U. R. \& Aubert, J. Scaling properties of convection-driven dynamos in rotating spherical shells and application to planetary magnetic fields. Geophys. J. Int. 166, 97-114 (2006).

\section{Acknowledgements}

We thank Francis Nimmo and Yohai Kaspi. E.T. thanks the Weizmann Institute for its hospitality during parts of this work. Y.A. was funded by the U.S-Israel Binational
Science Foundation (BSF grant number 2018152). E.T. was funded by the National Aeronautics and Space Administration Habitable Worlds programme (grant FP062796A/NNX16AR85G)

\section{Author contributions}

Y.A. and E.T. took part in all stages of the work.

\section{Competing interests}

The authors declare no competing interests.

\section{Additional information}

Supplementary information The online version contains supplementary material available at https://doi.org/10.1038/s41467-021-26710-0.

Correspondence and requests for materials should be addressed to Yosef Ashkenazy.

Peer review information Nature Communications thanks Christophe Gissinger, Jason Goodman and the other, anonymous, reviewer for their contribution to the peer review of this work.

Reprints and permission information is available at http://www.nature.com/reprints

Publisher's note Springer Nature remains neutral with regard to jurisdictional claims in published maps and institutional affiliations.

(c) (i) Open Access This article is licensed under a Creative Commons Attribution 4.0 International License, which permits use, sharing, adaptation, distribution and reproduction in any medium or format, as long as you give appropriate credit to the original author(s) and the source, provide a link to the Creative Commons license, and indicate if changes were made. The images or other third party material in this article are included in the article's Creative Commons license, unless indicated otherwise in a credit line to the material. If material is not included in the article's Creative Commons license and your intended use is not permitted by statutory regulation or exceeds the permitted use, you will need to obtain permission directly from the copyright holder. To view a copy of this license, visit http://creativecommons.org/ licenses/by/4.0/.

(C) The Author(s) 2021 